produced by the mechanism favoured by Jakosky et al. rather than groundwater discharge.

So what can one conclude from all this? The available observations cannot tell us if the material underlying the residual north cap is water ice or dry ice. Nor can they tell us if oceans were present during the past several billion years, or if Mars did have a substantial $\mathrm{CO}_{2}$ atmosphere. What they do tell us is that Mars was different in the past, and that somehow water was able to flow across its surface. But until we return to Mars and take the next step in the exploration of this fascinating planet, all we can do is speculate.

Robert M. Haberle is in the Space Science Division, NASA Ames Research Center, Moffett Field, California 94035, USA.

\section{What is remembered and why?}

\section{AlexanderM. Klibanov}

Molecular memory is the curious phenomenon whereby functional properties of a substance depend on the sample's history. For example, when methacrylic acid and ethylene glycol dimethacrylate are copolymerized in the presence of the bronchodilator theophylline and then the latter is washed out, the resultant crosslinked polymer is capable of binding this drug; the same polymer prepared in the absence of theophylline under otherwise identical conditions cannot ${ }^{1}$. Ever since the first report of enzyme memory in organic solvents ${ }^{2}$, questions have persisted about its mechanism. Now, X-ray crystallography by Yennawar et al. ${ }^{3}$ has shed some light on this issue.

The original observation ${ }^{2}$ was that lyophilization of the protease subtilisin from aqueous solution containing various competitive inhibitors (followed by their removal by anhydrous extraction) created an enzyme which was up to 100 times more active in anhydrous solvents than the enzyme lyophilized in the absence of ligands. This ligand-induced enzyme memory extended to the stability, affinity and substrate specificity of subtilisin in organic solvents, but it vanished when the enzyme was redissolved in water. Ståhl et $a l .{ }^{4}$ subsequently demonstrated that the enantioselectivity of another protease, chymotrypsin, in organic solvents was also markedly affected by the chirality of the ligand present in aqueous solution of the enzyme during dehydration.

To rationalize these findings, it was hypothesized $^{2,4}$ that the ligands caused conformational changes in the enzyme molecules and that even after removal of the ligand such 'imprints' were retained by the enzyme in anhydrous media because of its rigidity in the absence of water ${ }^{5}$. Because the structures of ligandimprinted enzymes are distinct from those of their non-imprinted counterparts, so are the catalytic properties.

To assess this, Yennawar et al..$^{3}$ soaked crystals of chymotrypsin - the same enzyme studied by Ståhl et al. - in hexane containing the enzyme inhibitor $N$-acetylD-tryptophan. Although the inhibitor 596 bound to the active site of chymotrypsin in this solvent, they found that the X-ray crystal structure of the enzyme in the complex was essentially identical to that of the free chymotrypsin in hexane ${ }^{6}$.

The very fact that enzyme studies in aqueous solutions are reproducible - that given characteristics (activity, $\mathrm{pH}$-profile, stability and so on) of an enzyme are independent of where, when and by whom the measurements have been conducted - indicates that the behaviour of enzymes in water is invariant of their history. This lack of memory stems from conformational flexibility of enzymes in aqueous solution $^{7,8}$, where they are in a state of intensive perpetual motion or 'breathing'. Whatever reversible conformational changes, including ligand imprinting, may have occurred in the enzyme molecule beforehand are irrelevant, because in water the enzyme will shake them off and acquire the thermodynamically favoured conformation.

Thus one condition for enzyme memory, which is not met in water, is high conformational rigidity in the medium where the memory is to be displayed. The study by Yennawar et al. ${ }^{3}$ points to a further requirement - considerable conformational flexibility in the medium where the enzyme memory is induced by a ligand - which is met in water but not in hexane. On the other hand, both conditions are satisfied when an enzyme is imprinted with a ligand in water, followed by lyophilization and ligand removal, and then the memory is tested in anhydrous solvents ${ }^{2}$.

1. Vlatakis, G., Andersson, L. I., Muller, R. \& Mosbach, K. Nature 361, 645-647 (1993).

2. Russell, A. J. \& Klibanov. A. M. J. biol. Chem. 263, $11624-11626$ (1988).

3. Yennawar, H. P., Yennawar, N. H. \& Farber, G. K. J. Am. chem. Soc. 117, 577-585(1995).

4. Ståhl, M. etal. J. Am. chem. Soc. 113, 9366-9368 (1991).

5. Burke, P. A., Griffin, R. G. \& Klibanov, A. M. Biotechnol. Bioengng 42, 87-94 (1993).

6. Yennawar, N. H., Yennawar, H. P. \& Farber, G. K. Biochemistry 33, 7326-7336 (1994)

7. Karplus, M. \& McCammon, J. A. A. Rev. Biochem. 52. 263-300 (1983)

8. Rupley, J. A., Gratton, E. \& Careri, G. Trends biochem. Sci. 8, 18-22 (1983).
There is growing spectroscopic evidence that on lyophilization from aqueous solutions many proteins undergo profound, reversible, conformational changes $^{9-12}$. This denaturing can be prevented to varying degrees, however, by adding certain excipients (lyoprotectants) to protein solutions before lyophilization $^{11,12}$. These findings have expanded the range of chemical messages that enzymes remember in anhydrous solvents beyond active site-binding ligands ${ }^{2-4}$ to include certain sugars ${ }^{13}$ and salts $^{14}$ that bind to other parts of the enzyme molecule.

Enzymatic conversions in non-aqueous media broaden the utility of enzymes as practical catalysts, in particular for the synthesis of chiral pharmaceutical intermediates ${ }^{15}$. Imprinted enzymes hold a special promise because of their superior catalytic activity ${ }^{2,13,14}$ and even adjustable enantioselectivity ${ }^{4}$ in organic solvents. To take full advantage of this unique opportunity, much more must be learned about the strength and scope of enzyme memory, as well as the relationship between the nature of the imprinting excipient and the ensuing function of the enzyme preparation. Such knowledge can only come from structural investigations.

If enzyme crystals are to be used ${ }^{16}$ in organic solvents, the work of Yennawar et $a .^{3}$ shows that X-ray crystallographic studies should be instructive provided that the crystals are grown in water in the presence of a ligand, followed by replacement of water with an organic solvent containing the same ligand. Limitations of this strategy are the likely heterogeneity of the resultant enzyme populations and difficulties in crystallizing them.

Neither these limitations nor restrictions on the type of memory-inducing excipients exist with lyophilized enzymes in organic solvents ${ }^{2,4,13,14}$. Here such biophysical techniques as Raman and Fourier-transform infrared spectroscopies $^{10,11}$ can be informative, although additional methodologies for the structural analysis of amorphous protein samples are certainly needed.

Alexander M. Klibanov is in the Department of Chemistry, Massachusetts Institute of Technology, Cambridge, Massachusetts 02139, USA.

\footnotetext{
9. Poole, P. L. \& Finney, J. L. Biopolymers 22, 255-260 (1983).

10. Prestrelski, S. J., Arakawa, T. \& Carpenter, J. F. Arch. Biochem. Biophys. 303, 465-473(1993).

11. Prestrelski, S. J. etal. Biophys. J. 65, 661-671(1993)

12. Desai, U. R., Osterhout, J. J. \& Klibanov, A. M. J. Am. chem. Soc. 116, 9420 9422 (1994).

13. Dabulis, K. \& Klibanov, A. M. Biotechnol. Bioengng 41 566-571 (1993)

14. Khmeinitsky, Y. L., Welch, S. H., Clark, D. S. \& Dordick J.S.J. Am. chem. Soc. 116, 2647-2648 (1994).

15. Crout, D. H. G.. Roberts, S. M. \& Jones, J. B. (eds) Tetrahedron: Asymmetry 4 (spec. issue), 757-1080 (1993).

16. St Clair, N. L. \& Navia, M. A. J. Am. chem. Soc. 114 7314-7316 (1992).
}

NATURE · VOL 374 · 13 APRIL 1995 